\title{
Criminologie
}

\section{Historique des normes juridiques dans les pénitenciers au Canada}

\section{Lucie Lemonde}

Volume 28, numéro 1, 1995

L’histoire de l'enfermement et des populations pénales

URI : https://id.erudit.org/iderudit/017366ar

DOI : https://doi.org/10.7202/017366ar

Aller au sommaire du numéro

\section{Éditeur(s)}

Les Presses de l'Université de Montréal

ISSN

0316-0041 (imprimé)

1492-1367 (numérique)

Découvrir la revue

Citer cet article

Lemonde, L. (1995). Historique des normes juridiques dans les pénitenciers au Canada. Criminologie, 28(1), 97-117. https://doi.org/10.7202/017366ar
Résumé de l'article

The author brings forth three main periods in the evolution of legislative, statutory and administrative norms regarding the powers of the correctional authorities and the treatment of prisoners in Canadian penitentiaries, from 1834 to present. The first two periods are characterized by an extreme prescriptive profusion typical of utilitarian institutions and by the delegation of normative power to administrative authorities. During the 1980's, the intervention of the courts forces the legislature to progressively integrate the notion of respect for the rule of law and for the principles of procedural equity. The new Law on the correctional system and conditional release in effect since November 1992, is radically distinct from the types of legislations that prevailed for the preceeding fifty years in that it incorporates and ratifies the discourse on prisoners' rights. According to the author, the change of perspective is radical: the utilitarian function of carcéral norms has weakened at the benefit of a function protective of prisoners' rights.
Ce document est protégé par la loi sur le droit d'auteur. L'utilisation des services d’Érudit (y compris la reproduction) est assujettie à sa politique d'utilisation que vous pouvez consulter en ligne.

https://apropos.erudit.org/fr/usagers/politique-dutilisation/ 
The author brings forth three main periods in the evolution of legislative, statutory and administrative norms regarding the powers of the correctional authorities and the treatment of prisoners in Canadian penitentiaries, from 1834 to present. The first two periods are characterized by an extreme prescriptive profusion typical of utilitarian institutions and by the delegation of normative power to administrative authorities. During the 1980's, the intervention of the courts forces the legislature to progressively integrate the notion of respect for the rule of law and for the principles of procedural equity. The new Law on the correctional system and conditional release in effect since November 1992, is radically distinct from the types of legislations that prevailed for the preceeding fifty years in that it incorporates and ratifies the discourse on prisoners' rights. According to the author, the change of perspective is radical: the utilitarian function of carceral norms has weakened at the benefit of a function protective of prisoners' rights.

L'évolution des normes législatives et administratives régissant les pouvoirs des autorités carcérales et le traitement des prisonniers dans les pénitenciers canadiens jusqu'aux années 1980 présente toutes les caractéristiques des règles propres aux institutions totalitaires telles que décrites par les analystes de l'enfermement ou de la prison ${ }^{2}$ : profusion normative extrême, régime disciplinaire autonome et très strict visant la soumission et la reproduction du modèle, contrôle total sur la vie, la liberté et la sécurité des détenus, système de privilèges institutionnalisé, négation des droits et de la zone d'autonomie des prisonniers, pouvoir discrétionnaire absolu et non encadré, absence de contrainte légale formelle, etc.

1. Professeure, Département des sciences juridiques, Université du Québec à Montréal, C.P. 8888, Succ. centre-ville, Montréal, Québec, H3C 3P8

2. Erving GOFFMAN, Asiles, Paris, Les Éditions de Minuit, 1968, 447 p.; D. J. ROTHMAN, The Discovery of the Asylum: Social Order and Disorder in the New Republic, Boston, Little, Brown and Co., 1971 ; Gresham SYKES, The Society of Captives, Princeton, Princeton University Press, 1958; Michel FOUCAULT, Surveiller et punir. Naissance de la prison, Paris, Gallimard, 1975, 318 p.; Danièle LOSCHAK, «Droit et non-droit dans les institutions totalitaires. Le droit à l'épreuve du totalitarisme ", L'Institution, C.U.R.A.P.P., Paris, P.U.F., 1981, pp. 125-184. 
L'analyse du contenu des premières législations, réglementations et règles administratives en vigueur dans les pénitenciers canadiens démontre que, jusqu'à tout rêcemment, la normativité interne ne retenait que la fonction instrumentaliste du droit et en rejetait la dimension de protection contre le pouvoir. La majorité des règles, de nature répressive plutôt que permissive, consistait en des interdictions et des devoirs assortis de sanctions. Les normes et les principes libéraux du droit en vigueur dans la société globale n'avaient pas cours dans l'ordre juridique carcéral.

C'est dans le cadre d'une institution totalitaire «sans droit qu'a émęrgé depuis quelques années une normativité fondée sur les principes d'éthique procédurale et sur les droits fondamentaux. La nouvelle Loi sur le système correctionnel et la mise en liberté sous condition ${ }^{3}$, en vigueur depuis novembre 1992, se démarque radicalement du type de législations ayant prévalu au cours des cent cinquante années précédentes en ce qu'elle intègre et consacre le discours des droits des personnes incarcérées.

\section{LA FONCTION DU DROIT DANS L'INSTITUTION TOTALITAIRE}

La principale caractéristique d'une institution totalitaire, selon Goffman, est une vie coupée du monde extérieur dont les modalités sont minutieusement réglées par un règlement intérieur précisant le détail des interdictions et prescriptions auxquelles les reclus doivent se plier. En second lieu, on y retrouve un système de gratifications et sanctions, c'est-à-dire d'une part des récompenses et faveurs accordées en échange de la soumission au personnel et, d'autre part, les punitions, c'est-à-dire le retrait de ces faveurs ou la suppression du droit de les briguer ${ }^{4}$. Foucault, pour sa part, parle de la prison comme d'une institution "complète et austère» qui a ses mécanismes internes de répression ${ }^{5}$ et qui n'est pas soumise aux règles de la vie sociale normale mais fonctionne de façon tout à fait autonome, invisible et souveraine ${ }^{6}$.

La discipline est l'élément central de toute institution totalitaire. Cette dernière ne vise pas uniquement le maintien de l'ordre et le respect du règlement, mais son but avoué est de transformer le coupable en un être soumis respectueux des lois $^{7}$. La discipline carcérale est gérée de façon

3. S.C. 1992 , c. 20.

4. E. GOFFMAN, Asiles, op. cit., note 1, p. 41.

5. M. FOUCAULT, Surveiller et punir, op. cit., note 1, p. 238.

6. C'est ce que Foucault a appelé la Déclaration d'indépendance carcérale, Ibid., p. 250.

7. Selon l'article 38 de la Loi sur le système correctionnel et la mise en liberté sous condition, précitée, note 2 , le régime disciplinaire vise à encourager chez les détenus un comportement favorisant l'ordre et la bonne marche du pénitencier, tout en contribuant à leur réadaptation et à leur réhabilitation sociale à titre de citoyens respectueux des lois. 
autonome par rapport à l'ordre juridique global et par rapport au pouvoir judiciaire: elle crée ses propres délits, ses propres sanctions, ses propres règles de preuve et ses propres instances décisionnelles ${ }^{8}$.

Dans l'ordre juridique carcéral, les règles autoritaires sont omniprésentes. En plus de la législation-cadre et du règlement interne, il existe une foule de directives, d'instructions, d'ordres, de notes de service, de manuels de conduite destinés aux détenus, etc. Ces textes régissent minutieusement les moindres aspects de la vie quotidienne. Malgré cette profusion normative, presque tous les analystes des systèmes pénitentiaires concluent qu'il s'agit d'États sans droit ${ }^{9}$. Le professeur Loschak a très bien identifié cette contradiction fondamentale en se demandant pourquoi l'institution carcérale, si fortement structurée juridiquement, apparaît comme un espace de non-droit et le lieu par excellence de l'arbitraire. Selon elle, si «nous parvenons mal à percevoir l'institution totalitaire comme un univers de droit, en dépit de la normativité extrême qui s'y déploie ", c'est peut-être «parce que la dégénérescence de la forme juridique ne laisse subsister que les signes extérieurs de la règle de droit et en dissout l'essence, la substance protectrice $^{10} »$.

Selon elle, "l'ordre juridique totalitaire ne rejette pas la notion de droit mais il n'en retient que la fonction instrumentaliste ". L'équilibre que l'on retrouve habituellement dans la société libérale entre le droit comme instrument du pouvoir et le droit comme arme contre le pouvoir n'existe plus dans l'ordre totalitaire. La notion de réciprocité n'existe pas dans les rapports juridiques au sein de l'institution carcérale et les prérogatives du pouvoir l'emportent définitivement sur les droits des individus " ${ }^{11}$ Pendant longtemps, on a affirmé que les prisonniers ne jouissaient d'aucun droit civil à l'exception de ceux qui leur étaient spécifiquement reconnus par la loi.

8. "Au cœur de tous les systèmes disciplinaires, fonctionne un petit mécanisme pénal (...) avec ses lois propres, ses délits spécifiés, ses formes particulières de sanction, ses instances de jugement. " M. FOUCAULT, Surveiller et punir, op. cit., note 2, p. 180.

9. David GREENBERG et Fay STENDER, "The Prison as a Lawiess Agency ", (1972) Buffalo Law Review, vol. 22, p. 799 ; Michael JACKSON, «The Right to Counsel in Prison Disciplinary Hearings", (1986) University of British Columbia Law Review, vol. 20, 221, 221; voir aussi, M. JACKSON, "Justice Behind the Walls - A Study of the Disciplinary Process in Canadian Penitentiary " (1974), Osgoode Hall Law Journal, vol, 12, 1.

10. D. LOSCHAK, « Droit et non-droit dans les institutions totalitaires », loc. cit., note 1, p. 155 .

11. Ibid., p. 175. Voir aussi Erik Olin WRIGHT (éd.), The Politics of Punishment : A Critical Analysis of Prisons in America, New York, Harper and Row Pubs, 1973. 
Avant que certaines de ces spécificités ne commencent à s'affaiblir et que n'émerge, au cours des années 1980 , une normativité des droits fondamentaux fondée sur le respect de la règle de droit et que les tribunaux ne forcent le législateur à intégrer des principes d'éthique procêdurale, il est possible d'identifier deux grandes périodes, l'une allant de 1834 à 1960, se caractérisant par une profusion normative répressive propre aux institutions totalitaires, et l'autre, de 1960 à 1979, se distinguant par une abdication législative notable et par une délégation du pouvoir normatif aux autorités administratives.

\section{LA PROFUSION NORMATIVE DANS L'INSTITUTION TOTALITAIRE (1834-1960)}

Les premières législations concernant l'incarcération des criminels au Canada se caractérisent par la dévolution des pleins pouvoirs au directeur de l'institution et par l'absence de contrôle étatique véritable sur l'administration pénitentiaire. Ces lois volumineuses s'attardent avec force détails sur la tâche, la conduite et les émoluments des officiers au service du pénitencier mais sont plutôt laconiques sur le traitement des prisonniers. La notion de droits des personnes incarcérées est tout à fait inexistante. Les législations sont complétées par des instructions et des règles émises par les inspecteurs des pénitenciers dans lesquelles se retrouve une profusion de normes régissant les moindres aspects de la vie institutionnelle carcérale.

\section{A. Les déclarations de principes et énoncés de mission.}

Au XIX siècle, les nombreuses législations concernant les pénitenciers $^{12}$ contiennent quelques grandes déclarations de principes pénologiques et moraux répandues à l'époque. Les concepts-clés de la philosophie

12. Nous en avons dénombré onze entre 1834 et 1886: Act to provide for the Maintenance and Government of the Provincial Penitentiary, erected near Kingston, in the Midland district, S.C., 1834, 4 Will. IV, c. 37; Act to render the Penitentiary erected near Kingston, in the Midland district, the Provincial Penitentiary for Canada, S.C., 1841,4 \& 5 Vict. c. 69 . Act for better proportioning the punishment to the offence, in certain cases, and for other purposes therein mentioned, S.C., 1842, 6 Vict. c. $5 ;$ Act to consolidate and amend the Laws to the Provincial Penitentiary, S.C., 1846, 9 Vict. c. 4; An Act for the better Management of the Provincial Penitentiary, S.C., 1851, 14 \& 15 Vict. c. 2; Acte concernant les inspecteurs des asiles et des hôpitaux publics, du penitentiaire provincial et de toutes les prisons communes et autres prisons, S.C., 1859, 22 Vict. c. 110; Acte concernant le pénitentiaire provincial, S.C., 1859, 22 Vict. c. 111 ; Acte concernant les pénitenciers et les directeurs préposés à leur administration, et pour d'autres fins, S.C., 1868, 31 Vict. c. 75 ; Acte concernant les pénitenciers et leur inspection, et pour d'autres fins, S.C., 1875, 38 Vict. c. 44; Acte pour amender et refondre la législation sur les pénitenciers, S.C., 1883, 46 Vict., c. 37; Acte concernant les pénitenciers, S.R.C., 1886, c. 182. 
de ces premières législations sur l'incarcération pénitentiaire sont la réforme, la dissuasion, la réclusion solitaire, la discipline, l'instruction religieuse et les travaux forcés. Tout le langage employé reproduit cette philosophie.

Ainsi, la toute première loi prévoyant l'érection du premier pénitencier à Kingston au Canada ${ }^{13}$ reprend le préambule de la Loi anglaise de $1779^{14}$, proclamant que : " [If] many offenders convicted of crimes were ordered to solitary imprisonment, accompanied by well regulated labor and religious instruction, it might be the means under Providence, not only of deterring others from the commission of like crimes, but also reforming the individuals, and inuring them to habits of industry. "

La loi de 1851 affirme elle aussi la fin réformatrice des pénitenciers lorsqu'elle énonce que le directeur doit rechercher "the success of the religious, moral and industrial appliances used for the reformation of the convicts ${ }^{15}$ ". Elle spécifie aussi que les inspecteurs doivent, dans leur rapport annuel, "make special reference to the moral effects of discipline upon convicts». L'aumônier du pénitencier est tenu, pour sa part, d'administrer aux prisonniers des exhortations morales "calculated to promote their spiritual welfare, moral reformation and due subordination $^{16}$ ".

La loi promulguée en 1868 exprime très bien ces orientations, principalement dans le considérant de l'article 61 appuyant la demande de construction de cellules dites "pénales", c'est-à-dire de cellules couplées chacune à un atelier pour mener à bien le système de discipline séparé et solitaire :

Considérant qu'aucun système de discipline ne peut être efficace dans un pénitencier pour la punition ou la réforme des criminels, s'il n'est combiné avec une réclusion solitaire rigoureuse pendant une certaine période du temps pour laquelle la cour l'aura condamné à être emprisonné, et qu'il est en conséquence nécessaire de prendre les mesures dans tous les pénitenciers désignés, ainsi que tous les autres qui le seront dans l'avenir, pour effectuer

13. Act to provide for the Maintenance and Government of the Provincial Penitentiary, erected near Kingston, in the Midland district, precité, note 12.

14. Penitentiary Act, (1779) Geo. III, c. 4.

15. An Act for the Better Management of the Provincial Penitentiary, precite, note 11 , art XV.

16. Ibid, art. XVII. Il doit aussi, précise la disposition, éviter avec soin d'encourager les plaintes des détenus contre leurs gardiens ou contre leur traitement et ne pas leur communiquer d'informations autres que religieuses. 
la réclusion solitaire de chaque détenu pendant une certaine période du temps mentionné dans la sentence ${ }^{17}$.

Les quelque onze lois concernant les pénitenciers adoptées entre 1834 et 1906 contiennent des dispositions voulant que tous les prisonniers soient constamment employés aux travaux forcés pendant le jour pour le bénéfice du pénitencier, tous les jours de l'année, à l'exception de quelques fêtes religieuses ${ }^{18}$. On indique également qu'il est du devoir du directeur de garder chaque détenu seul dans une cellule durant la nuit ou durant le jour sauf pour le travail et que, lorsque les détenus sont rassemblés dans les ateliers ou autres lieux de travail, le directeur doit les maintenir aussi séparés que possible, ne leur permettant aucune autre relation ou conversation que celles absolument requises pour le travail ${ }^{19}$.

Conformément aux courants philosophiques régnant en Europe et aux États-Unis, l'on semble convaincu au Canada que l'isolement, le silence complet, la discipline de fer et l'emploi constant aux travaux forcés vont, d'une part, réformer les criminels et, d'autre part, constituer un régime de vie si pénible qu'il dissuadera les gens à tout jamais de commettre des crimes. Or, très tôt, on se rend compte que ce n'est pas le cas puisque plusieurs prisonniers récidivent et, de fait, reviennent au pénitencier. Dans son rapport annuel en 1840, l'aumônier de Kingston déplore le fait que les sentences imposées soient trop courtes parce que, affirme-t-il, il n'est pas possible dans un si court laps de temps de véritablement réformer et changer le cour d'un crimine ${ }^{20}$. Cette recommandation semble être à l'origine de la distinction prévalant encore de nos jours entre les sentences de plus de deux ans et celles de moins de deux ans comme fondement du lieu de l'incarcération dans un pénitencier plutôt que dans une prison provinciale ${ }^{2 !}$.

17. Acte concernant les pénitenciers et les directeurs préposés à leur administration, précité, note 11. Ce considérant sera abandonné en 1886.

18. Le travail forcé des prisonniers a continue à être la norme au Canada bien après cette date. Si l'emploi des termes "travaux forcés» a disparu dans la législation à partir de 1960, les prisonniers sont toujours astreints au travail. L'article 40 p) de la loi actuelle édicte que le refus de travailler ou l'absence au travail sans excuse valable constitue une infraction disciplinaire. Voir Loi sur le systeme correctionnel et la mise en liberté sous condition, précitée, note 3.

19. La règle du silence absolu de même que celle de l'isolement pendant le jour sont abandonnées en 1906. Voir Loi concernant les pénitenciers, S.R.C., 1906, c. 147, art. 65 et 61 .

20. Chaplain's Report to the Inspectors of the Provincial Penitentiary, Appendix to Journal of the House of Assembly of Upper Canada, 5th Ses., 13th Parl., 3rd Vic. 1840.

21. C'est en effet en 1842 qu'apparaît pour la première fois dans la législation canadienne cette distinction entre les sentences de deux ans et plus: Act for better proportioning the punishment to the offence, in certain cases, and for other purposes therein mentioned, précité, note 12 . 
La pratique de formuler dans la loi des déclarations de principe et énoncés de mission devant gouverner l'action et l'esprit des autorités pénitentiaires dans le traitement des prisonniers a été abandonnée à la fin du siècle dernier. Elle réapparaîtra cent ans plus tard dans la Loi sur le système correctionnel et la mise en liberté sous condition ${ }^{22}$.

\section{B. L'administration pénitentiaire}

L'organisation de l'administration pénitentiaire est demeurée très simple et très peu contrôlée ou encadrée par le pouvoir exécutif jusqu'en 1960. Les premières législations sont muettes sur l'identité de l'autorité gouvernementale responsable des pénitenciers. On y précise uniquement que les inspecteurs et directeurs sont nommés par le gouverneur général et qu'ils doivent faire rapport au gouverneur général en conseil. À compter de $1875^{23}$, les pénitenciers sont sous le contrôle du ministre de la Justice dont les inspecteurs sont les représentants. En $1939^{24}$, une Commission des pénitenciers est établie pour la première fois. Elle est composée de trois commissaires nommés pour dix ans par le gouverneur en conseil et elle doit édicter des règles et règlements, relevant du ministre, concernant la gestion, la discipline, 1'administration et le travail dans les pénitenciers. En $1952^{25}$, cette commission est dirigée par un seul commissaire assisté de deux souscommissaires, tous trois amovibles.

Pendant toutes ces années, l'ensemble du pouvoir exécutif est confié au directeur (ou préfet) alors que le pouvoir d'établir des règles ou instructions et de surveiller l'administration générale des pénitenciers relève des inspecteurs (ou surintendants ou, plus tard, commissaires).

Les législations prévoient en effet que le ou les pénitenciers sont dirigés par des inspecteurs nommés par le gouverneur général. Le nombre d'inspecteurs a varié au cours des années, allant de deux à cinq, puis de nouveau un seul que l'on nommera par la suite commissaire des pénitenciers. Ces inspecteurs n'ont pas de pouvoir exécutif direct sauf de donner des instructions aux directeurs sur la conduite de l'institution. Ils peuvent établir des règles ou règlements sur la discipline, l'administration, la conduite des directeurs et des employés, l'alimentation, l'emploi, l'instruction, la discipline et la correction, les châtiments et les récompenses des détenus. À partir de 1868 , ces règles doivent recevoir l'approbation du gouverneur en conseil.

22. Précité, note 3.

23. Acte concernant les pénitenciers et leur inspection, et pour d'autres fins, précité, note 12 .

24. Loi concernant les pénitenciers, S.C., 1939, c. 6.

25. Loi concernant les pénitenciers, S.R.C., 1952, c. 206. 
Les inspecteurs doivent aussi enquêter sur la régie et les finances du pénitencier, le travail, la tenue de livres, vérifier l'ordre, la propreté, la qualité de la nourriture et s'assurer que les offenses sont sagement et humainement châtiées. Ces inspecteurs, à part le devoir de visiter fréquemment le pénitencier, de tenir les minutes de leurs visites et de faire un rapport annuel au gouverneur général en conseil, n'ont aucune autre obligation de rendre compte au Parlement ${ }^{26}$.

Le directeur a le contrôle exclusif du pénitencier. Il doit veiller à la santé, l'alimentation, la morale, la conduite et la garde des prisonniers et s'assurer que la justice, la bienveillance et la moralité prévalent dans l'institution. Il est responsable des contrats et des registres et doit tenir un journal quotidien sur l'inconduite ou la négligence des officiers, sur les plaintes bien fondées des prinonniers concernant la nourriture ou les traitements cruels ou inusités. Le directeur ou préfet forme, à lui seul, une corporation et tous les contrats sont faits à son nom. Il perçoit lui-même les amendes à certaines infractions réglementaires.

Jusqu'en 1960, les diverses lois confient aux directeurs l'entière administration et le contrôle exécutif de la prison, sans autre obligation que de se conformer aux règles dûment établies et aux instructions écrites des inspecteurs et du ministre.

Les premières lois décrivent précisément les devoirs et les pouvoirs de chacun des officiers à l'emploi du pénitencier, à partir du directeur jusqu'au garde en passant par le clerc, le médecin, l'aumônier, le magasinier, etc. La majeure partie de ces législations concernant les pénitenciers est consacrée non pas aux prisonniers, mais aux employés, allant même jusqu'à établir dans les moindres détails les salaires et autres avantages de chaque catégorie d'emplois.

\section{La vie carcérale et le traitement des prisonniers}

\section{i) Les «droits» des prisonniers}

Bien que les premières législations soient très volumineuses, seules quelques dispositions concernent spécifiquement le traitement des prisonniers. La loi de 1834 ordonne que ceux-ci soient vêtus en matériel brut manufacturé au pénitencier et qu'ils soient nourris «with sufficient quantity of inferior and wholesome food». On doit aussi leur fournir des

26. Dans le Rapport de la Commission royale d'enquête sur le système pénal du Canada, Ottawa, 1938 (Rapport Archambault), les commissaires critiquent fortement le fait que le surintendant des pénitenciers régisse tout de façon arrogante, ne laissant aucun pouvoir administratif au directeur. Ils soulignent également qu'au cours des ans, les divers inspecteurs ne se donnent même pas la peine, sauf exception, de visiter les pénitenciers tel que requis par la loi. 
médicaments et une Bible. Le seul autre droit reconnu au prisonnier est celui de ne pas être obligé de quitter le pénitencier entre les mois de novembre et de mars et de recevoir, au moment de sa libération, une petite somme d'argent et des vêtements.

L'article 31 de la loi de 1868 énumère les règles à suivre pour le traitement des détenus. Il faut noter que ces prescriptions ne sont pas exprimées en termes de droits pour les prisonniers mais en termes d'obligations pour le directeur :

1. Pendant la durée de son emprisonnement, chaque détenu sera vêtu, aux frais du pénitencier, de l'uniforme destiné aux prisonniers ;

2. Sa nourriture se composera d'aliments sains qui lui seront servis en quantité suffisante;

3. Il aura un lit, un oreiller et des couvertures suffisantes variant selon la saison;

4. Excepté en temps de maladie ou autre incapacité, il sera constamment employé aux travaux forcés, dont la nature sera déterminée par le préfet, - chaque jour ne devant pas excéder dix heures à part le temps consacré aux repas $[\ldots]$;

5. Nul détenu catholique romain ne sera contraint de travailler pendant les fêtes d'obligation établies par son Église [...];

8. Sauf les cas de maladie, chaque détenu sera, pendant la nuit, enfermé seul dans une cellule, et aussi pendant le jour s'il n'est pas employé.

À partir de 1868 , un régime de remise de peine pour bonne conduite est établi et il relève de l'entière discrétion du directeur. L'article 62 de la Loi $^{27}$ énonce que :

Dans le but d'encourager les détenus à bien se conduire et à travailler avec soin et diligence, et de les en récompenser, il sera et pourra être loisible aux directeurs [...] de tenir un registre exact de la conduite journalière de chaque détenu d'un pénitencier, dans lequel il sera pris note de son industrie, de sa diligence et de son assiduité dans l'accomplissement de son travail, et de l'exactitude avec laquelle il observe le règlement de la prison, afin de permettre à ce détenu de mériter la remise du temps pour lequel il aura été condamné à l'emprisonnement, n'excédant pas cinq jours par mois durant lequel il aura tenu une conduite exemplaire [...].

Ce régime de remise de peine pour conduite exemplaire subira plusieurs modifications aux cours des ans, notamment au niveau du nombre de jours qu'il est possible d'accumuler. On prévoira également que la commis sion de certaines infractions, comme l'évasion et l'assaut sur un officier,

27. Acte concernant les pénitenciers et les directeurs préposés à leur administration, précité, note 12 . 
entraînent, en plus de la sentence imposée, la déchéance automatique de cette remise de peine.

\section{ii) Les interdictions}

Pour le reste, les législations ne prescrivent que des interdictions, des infractions et des punitions. Les interdictions sont nombreuses : interdiction de recevoir des visites, des colis ou des lettres, interdiction de parler aux gardiens ou aux autres détenus, interdiction de posséder des objets personnels, du tabac ou des spiritueux. La loi énumère quelques infractions dont la conduite violente, la tentative d'évasion ou la désobéissance à tout ordre légal et les sentences afférentes à chacune d'elles. Elle spécifie que l'on doit placer en isolement cellulaire les prisonniers qui ont une conduite réfractaire, que les châtiments corporels ne doivent être appliqués que dans les cas extrêmes et que les offenses ne peuvent pas être punies par plus de soixante-quinze (75) coups de fouet chacune, et ce en présence du directeur et du médecin.

\section{iii) Le règlement}

C'est dans le Règlement émis par l'inspecteur des pénitenciers en $1899^{28}$, puis dans celui de $1933^{29}$ que l'on retrouve le plus de détails sur la vie carcérale et le régime disciplinaire ${ }^{30}$. La profusion normative atteindra son point culminant entre les années 1930 et 1960 . Le surintendant nommé en 1932, le général D. M. Ormond, ancien colonel de l'armée, introduit dans les pénitenciers un régime de contrôle militaire très sévère et publie en 1933 un règlement de quelque 725 articles et dix annexes. Il rédigera par la suite 858 circulaires dont certaines contiennent 56 paragraphes, ainsi que diverses brochures ou lettres commentant, interprétant et souvent embrouillant ces règles déjà nombreuses. Cette abondance de normes réglementaires est fortement critiquée en 1938 dans le rapport de la Commission royale d'enquête sur le système pénal du Canada, comme étant peu mûrie,

28. Penitentiary Regulations, Ottawa, Government Printing Bureau, 1899, soumis par l'inspecteur des pénitenciers en vertu de l'article 15 de la loi et approuvés par le ministre de la Justice.

29. Penitentiary Regulations, Ottawa, Imprimeur du Roi, 1933. Ce règlement sera modifié à plusieurs reprises par diverses circulaires ou instructions administratives et sera consolidé en 1958 dans un document intitule « Consolidation and condensation of Penitentiary Regulations 1933, Penitentiary Officers Handbook 1952, Circulars Letters Issued Since February 1, 1934 and Other Administrative Instructions ».

30. Ces règles des inspecteurs, appelées Règlement, ne sont pas adoptées par le gouverneur en conseil mais sont uniquement approuvées par le ministre. Le titre de la consolidation de 1958 laisse penser que ces règles sont les ancêtres des directives du commissaire plutôt qu'un règlement au sens strict du terme. 
inefficace et portant «bien souvent sur des questions de détail absolument triviales ${ }^{31} »$.

\section{iv) Les devoirs des prisonniers}

Le règlement de 1899 est très détaillé sur les devoirs du prisonnier : devoir de propreté, devoir de respect envers les officiers, devoir d'obéissance aux ordres, sans commentaire ou hésitation, devoir de ne pas quitter le groupe auquel il est attaché, devoir de garder le silence et de ne pas converser avec qui que ce soit, devoir de ne pas quitter son travail des yeux ou de regarder au loin quand un visiteur est présent, devoir d'aller à la chapelle catholique ou protestante.

À ces devoirs s'ajoute une liste d'interdictions impressionnante : interdiction d'écrire à un autre détenu ou de luj faire des signes, interdiction d'être deux ou plus hors de la vision ou de la portée d'oreille des gardes, interdiction d'entrer dans la cellule d'un autre, interdiction de siffler, de crier, de faire des clins d'œil, de posséder des objets n'appartenant pas à l'institution. À compter de 1933, le règlement est encore plus détaillé et contient plusieurs autres interdictions, devoirs et infractions.

\section{v) La routine quotidienne}

Comme le soulignent Goffman et Foucault, il est impressionnant de voir à quel point la vie dans l'institution totalitaire est réglementée dans les moindres détails. La réglementation en vigueur au Canada jusqu'en 1960 illustre bien ce point même si le Commissaire des pénitenciers, dans sa préface à la consolidation du règlement en 1952, souligne à l'intention des officiers: "In human affairs, no book of rules can ever be devised to take care of every situation. »

Les dix premières pages de ce règlement décrivent «les cérémonies d'admission ${ }^{32}$ " d'un prisonnier au pénitencier : fouille, dépouillement des effets personnels ${ }^{33}$, examen médical pour vérifier la présence de maladies contagieuses ou de défauts physiques ou mentaux, enregistrement de ses particularités physiques et de son curriculum vitæ, rasage des cheveux, prise de photographies et d'empreintes, octroi d'un numéro d'immatriculation, remise des vêtements institutionnels et de la liste des règles de conduite et des offenses disciplinaires de la prison. Selon Goffman, ces formalités d'admission dans l'institution totalitaire visent le dépouillement et la mortification de l'arrivant afin qu'il se « laisse niveler, homogénéiser

31. Rapport de la Commission royale d'enquête sur le système pénal du Canada, précité, note 26,31 .

32. Selon les termes de Goffman, Asiles, op. cit., note 2, p. 59.

33. À compter de 1938 , le règlement précise que le prisonnier peut conserver ses médailles, scapulaires et livres de prière. 
et transformer en un objet que l'on peut livrer à la machine de l'établissement de telle façon que rien ne vienne entraver la marche routinière des opérations administratives ${ }^{34}$ ».

Cette routine quotidienne est prévue dans les moindres détails. La prison ouvre au son de la cloche. Négliger de se lever promptement au premier son de la cloche le matin (ou de se mettre au lit au son de la cloche du coucher) constitue une infraction. Les détenus peuvent se parler d'un ton de voix normal de l'ouverture de la prison au son de cloche pour le travail, pendant le dîner jusqu'à la cloche du retour au travail, le soir et pendant les périodes de repos. Cependant, parler fort ou de choses obscènes est une offense. Se plaindre sans raison de la nourriture «dans le but évident de faire du trouble " constitue un bris de règles de discipline.

Les détenus doivent obéir aux ordres de se laver, prendre un bain ou se raser sous peine de sanction. Les hommes ne dojvent pas avoir les cheveux excédant un pouce et quart sur le front et le dessus de la tête et un pouce et demi sur les côtés et l'arrière de la tête. Ils ont droit à une période d'exercice en plein air de trente minutes par jour en hiver et de quarante minutes en été. Un tiers de cette période est réservé aux exercices rythmiques et systématiques. Le reste peut être consacré à des mouvements libres, mais ceux qui ont refusé de s'adonner aux exercices rythmiques ne peuvent participer aux exercices libres. S'il s'agit de jeux de groupe, comme le volley-ball, les prisonniers ne peuvent former d'alliances ou d'équipes naturelles. Il est permis de fumer, à la discrétion du directeur, pendant les dix minutes restantes.

Les prisonniers ont le droit d'écrire deux lettres par mois, mais chaque lettre ne doit traiter que d'un sujet à la fois. Tout ce qui parle de la conduite du pénitencier, de la sécurité et tout ce qui est contre l'intérêt public est censuré. Les détenus peuvent s'adonner à un hobby mais ils doivent, avant de commencer un dessin, obtenir du directeur l'approbation du sujet. Aucun livre, magazine ou journal controversé ou outrageant pour la foi religieuse n'est autorisé et le matériel de nature à entraîner l'esprit contre le bon ordre et la discipline, à enseigner des moyens de commettre un crime et à attenter à la morale, spécialement celle des détenus d'intelligence sous la normale, est interdit.

Les privilèges de visite sont aussi soigneusement précisés : sont autorisées à visiter les prisonniers : femme, enfants, père, mère, grands-parents, oncles, tantes, beaux-parents, etc. Les femmes détenues, quant à elles, ne peuvent recevoir de visiteurs mâles à l'exception de leur mari et de leurs fils s'ils sont âgés de moins de seize ans. Les visites sont autorisées à la discrétion du directeur une fois par mois pour une durée d'une demi-heure.

34. E. GOFFMAN, Asiles, op. cit., note 2, p. 59. 
En 1952, on autorisera, par amendement au règlement, les détenus à avoir des photographies «non-jmmorales » des personnes autorisées à les visiter ou encore de paysages.

Toutes ces normes sont formulées comme des privilèges et des gratifications-sanctions. Tout écart de conduite peut mener à la suppression de ces privilèges.

\section{vi) Le régime disciplinaire}

L'article 163 du règlement de 1933 énumère quelque vingt-neuf infractions à la discipline pénitentiaire. Plusieurs de ces infractions sont très vagues et couvrent à peu près tout geste de la vie quotidienne : manquer de respect envers un officier, enfreindre un ordre quelconque de tout officier, chanter, siffler ou faire tout bruit inutile, causer des ennuis sans raison, commettre un acte portant préjudice à autrui, flâner dans les galeries, faire signe à un autre prisonnier, être oisif, négligeant ou insouciant au travail, se conduire d'une manière indécente, négliger de fermer la porte de sa cellule après y être entré, négliger de se lever promptement au premier son de la cloche le matin ou de se mettre au lit au son de la cloche du coucher, troubler de façon quelconque le bon ordre et la discipline.

À cette liste, il faut joindre les vingt-cinq «Règles de conduite ou délits de prison" que l'on retrouve à l'Annexe I du règlement. Celles-ci sont exprimées en termes de devoirs pour le prisonnier. Ces devoirs sont pour la plupart l'envers des infractions énumérées à l'article 163, mais certains sont décrits avec encore plus de détails. On prévoit par exemple que le détenu ne doit pas converser avec les officiers et que, $s$ 'il y est obligé pour son travail ou autre raison, il « doit se tenir au garde à vous et l'appeler "Monsieur" ".

Le manquement à ces devoirs ou le non-respect de ces interdictions de même que toute conduite indécente ou impropre, toute négligence ou oisiveté au travail sont passibles de punition. La liste des sanctions est établie aux articles 179 et suivants du règlement de 1899. Les sanctions possibles sont le régime au pain et à l'eau pour un maximum de vingt et un repas consécutifs (le maximum est de six repas pour les femmes), le lit dur pour un maximum d'un mois, les boulets, les chaînes et les Oregon boots, l'isolement cellulaire, la flagellation avec la palette de cuir (qui a remplacé le chat-à neuf-queues), la mise aux fers en cellule, l'application du boyau d'arrosage, la perte de rémission de peine.

En 1933, la liste des punitions diffère légèrement. Les sanctions peuvent consister en la suppression de divers privilèges (dont ceux de fumer, de converser, d'aller à la bibliothèque, de recevoir des visiteurs, d'écrire des lettres), la perte de rémission de peine, le lit dur, le régime $n^{\circ} 1$ pour neuf repas (un livre de pain par jour et de l'eau), le régime $n^{\circ} 2$ (8 onces de 
pain par repas et une pinte de porridge contenant trois onces d'avoine et huit onces de patates le midi) pour vingt et un jours, l'emprisonnement aux cellules d'isolation. Pour certains délits précis, tels les actes de violence, la révolte, l'insurrection, le langage très insultant envers un officier, le directeur peut ordonner, en plus de toute autre punition, que le prisonnier soit fouetté ou frappé à coups de courroie.

La profusion normative, principalement au niveau de la routine quotidienne et du régime disciplinaire, fournit aux autorités carcérales tous les outils nécessaires pour traiter les pensionnajres de l'institution totalitaire de façon discrétionnaire dans l'arbitraire le plus absolu. Ce pouvoir s'exerce dans l'ombre sans aucun contrôle externe. Comme l'a souligné Loschak, cet ordre juridique parallèle ne retient que la fonction instrumentaliste du droit et en dissout la substance protectrice. Cette constatation se vérifiera encore au cours de la prochaine période, avec quelques petites nuances toutefois.

\section{L'ABDICATION LÉGISLATIVE ET LA DÉLÉGATION DU POUVOIR NORMATIF (1960-1979)}

Au cours de la seconde période, l'administration des pénitenciers est plus centralisée. À compter de 1960, la Commission des pénitenciers devient le Service canadien des pénitenciers, dont l'administration centrale est située à Ottawa. Il est dirigé par le commissaire des pénitenciers qui décide de l'emplacement des administrations régionales dont les directeurs sont nommés par le ministre. À partir de 1966, le Service relève du ministère du Solliciteur général ${ }^{35}$.

\section{A. L'abdication législative}

La période allant de 1960 à 1979 se caractérise par une abdication législative notable. Tant les lois de $1960^{36}$ et de $1970^{37}$ que les deux règlements édictés en vertu de ces $\operatorname{lois}^{38}$ ont des contenus très minces. Ainsi, les nouvelles législations ne comptent qu'une trentaine de dispositions qui constituent en réalité des règles techniques sur l'organisation du Service, le lieu de l'incarcération, la réduction statutaire de peine, le mécanisme de remise de peine pour bonne conduite, les absences temporaires et le transfèrement des jeunes détenus ou ceux atteints de maladie mentale. D'autre part, le règlement, qui, comme on l'a vu, comprenait auparavant plus de 750 articles, n'en contient plus que quarante.

35. Loi de 1966 sur l'organisation du gouvernement, S.C., 1966-67, c. 25, art. 37.

36. Loi concernant les pénitenciers, S.C. 1960-61, c. 53.

37. Loi concernant les pénitenciers, S.R.C. 1970, c. P-6.

38. Règlement sur le service des pénitenciers, DORS/62-90; Règlement sur le service des pénitenciers, 1978, C.R.C., c. 1251. 
Le principal objet de la législation est la création d'organismes administratifs auxquels est déléguée la quasi-totalité du pouvoir normatif. La législation est muette sur la discipline ou le traitement des prisonniers sauf pour spécifier que le gouverneur en conseil peut édicter des règlements à ce sujet et pour déléguer au commissaire des pénitenciers le pouvoir normatif réel. L'article 29 (3) de la loi spécifie en effet que le commissaire peut "établir des règles, connues sous le nom d'Instructions du commissaire, concernant l'organisation, l'entraînement, la discipline, l'efficacité, l'administration et la direction judicieuse du Service, ainsi que la garde, le traitement, la formation, l'emploi et la discipline des détenus et la direction judicieuse des pénitenciers ».

Le règlement sur les pénitenciers établit quelques règles très générales concernant le traitement des prisonniers. Il prévoit par exemple que le détenu doit être incarcéré dans l'institution la plus appropriée compte tenu du degré de surveillance jugé nécessaire, qu'il doit être vêtu et nourri convenablement, qu'il doit recevoir les soins médicaux et dentaires essentiels, qu'il a droit à une période quotidienne d'exercices en plein air, qu'il peut se livrer à des passe-temps ou à la lecture, qu'il est astreint à se livrer à un travail pour lequel il peut recevoir un salaire.

Le langage employé dans le règlement laisse place à une discrétion très vaste et renvoie, à chaque disposition, soit aux directives du commissaire, soit à la notion de privilège. Ainsi le détenu doit-il bénéficier des soins médicaux essentiels ou d'une période d'exercice en plein air, "conformément aux directives ". La censure de la correspondance et des visites doit être évitée «dans la mesure où cela est pratique " et « rien aux présentes ne doit être considéré comme limitant l'autorité du Commissaire de réglementer, ou du chef de l'institution d'ordonner, la censure de la correspondance ou la surveillance des visites selon les modalités tenues pour nécessaires ou utiles à la rééducation et à la réadaptation des détenus ou à la sécurité de l'institution ${ }^{39}$ ». La lecture et les passe-temps sont permis dans la mesure où ils ne sont pas susceptibles "d'exercer une influence défavorable sur le bon ordre de l'institution 40 ». Le commissaire doit «dans la mesure où cela est pratique, assurer à chaque détenu susceptible d'en bénéficier, une formation académique ou professionnelle, un travail productif et instructif $^{41} \ldots$ ». Il doit aussi offrir à tout détenu qui en fait la demande la possibilité raisonnable de s'entretenir avec lui s'il «semble avoir un grief véritable et paraît pouvoir en bénéficier ${ }^{42}$.

39. Article 2.18 du règlement de 1960 et 28 de celui de 1978 .

40. Article 2.21 du règlement de 1960 et 31 de celui de 1978 .

41. Article 2.10 du règlement de 1960 et 20 de celui de 1978 .

42. Article 22 du règlement de 1978. 
Les nombreuses références aux notions de privilèges et de pouvoir discrétionnaire, de même que l'emploi de termes très vagues comme "dans la mesure où cela est pratique ", font en sorte que ces règles ne peuvent, en aucun cas, servir de base de revendication d'un droit devant l'État ou les autorités administratives. Les détenus vivent au royaume de l'arbitraire et de la délégation de pouvoir: des ordres et des directives, des pratiques et des règles plutôt que des lois et des règlements définissent le cadre de leur vie. Les décisions qui affectent grandement les intérêts fondamentaux des prisonniers ne sont pas prises par le Parlement mais par une multiplicité d'autorités déléguées qui opèrent largement hors des limites du contrôle du Parlement ${ }^{43}$.

Le fait que le législateur ait abdiqué ses pouvoirs d'élaboration de normes en matière carcérale ne signifie aucunement que la profusion normative existant au cours de la période précédente ne soit plus une réalité dans le monde pénitentiaire pendant ces années. Il y a tout simplement eu un déplacement d'autorité en ce domaine. Les normes autrefois législatives ou réglementaires sont devenues des normes administratives, mais elles sont tout aussi abondantes.

En effet, en plus des nombreuses directives du commissaire établies en vertu de l'article 29 (3) de la loi, on retrouve des «instructions divisionnaires " établies par les directeurs des diverses divisions régionales ${ }^{44}$ et des "ordres permanents » édictés par les directeurs des différents pénitenciers comprenant toutes les règles propres à leur institution ${ }^{45}$.

Il s'agit d'une situation tout à fait particulière. D'une part, le législateur demeure muet et ne détermine aucune norme précise, sauf pour déléguer aux autorités administratives le pouvoir de créer ses propres règles de fonctionnement. D'autre part, les règles ainsi énoncées dans les directives, instructions ou ordres permanents n'ont pas le statut de Joi ni ne lient les autorités qui les ont édictées. L'abdication législative est totale, et les quelques modifications législatives survenues au cours des ans sont des amendements techniques ou encore des réponses à la demande répétée des autorités de renforcer leur pouvoir discrétionnaire.

\section{B. Le régime disciplinaire}

Concernant le régime disciplinaire, le règlement établit une liste d'infractions (et de sanctions spécifiques) dont plusieurs seraient tout à fait inconcevables dans le monde libre, comme celles de gaspiller de la

43. Martha JACKMAN, «Case Comments. Fundamental Interests and Fundamental Justice. The Right to Participate in Decision-Making in Canadian Prisons : Piché c. S.-G. of Canada ", (1990) 24 University of British Columbia Law Rev. p. 361.

44. Article 1.14 du règlement de 1960 et 7 de celui de 1978 .

45. Article 1.15 du règlement de 1960 et 8 de celui de 1978 . 
nourriture ou de ne pas travailler de son mieux. De plus, plusieurs infractions à la discipline carcérale sont secrètes et non communiquées aux prisonniers qui doivent, tout en les ignorant, s'y soumettre sous peine d'être punis.

La liste des infractions disciplinaires, telle qu'édictée dans le règlement sur les pénitenciers, non seulement contient-elle certaines offenses dont la nature est très vague et imprécise, comme celle de commettre «un acte de nature à nuire à la discipline ou au bon ordre de l'institution ", mais elle viole aussi un principe très important du droit pénal voulant qu'il n'y ait «ni peine, ni crime sans loi ».

Le Règlement prévoit que le détenu commet une infraction disciplinaire s'il « enfreint quelque règlement, règle ou directive établis en vertu de la Loi ». Or, ces diverses règles se retrouvent dans des directives ou des instructions non-disponibles ou dans des «ordres permanents à propos desquels on spécifie expressément que les prisonniers ne doivent y avoir accès en aucune circonstance. Le fait que les détenus ne soient pas avisés de la conduite à laquelle ils doivent se conformer sous peine de sanction est non seulement contraire aux principes de justice élémentaire, mais a pour effet, comme Jackson le souligne avec justesse, «to further alienate and reinforce inmates' view of authority as illegitimate ${ }^{46}$ ».

Le processus disciplinaire de même que les processus décisionnels entourant les transfèrements disciplinaires ou les placements en ségrégation sont aussi tout à fait discrétionnaires et non conformes au modèle de due process ou au modèle de justice criminelle.

C'est au directeur de l'institution qu'incombent le devoir de maintenir la discipline et le pouvoir de punir. Aucune garantie procédurale n'est prévue dans le règlement. Les notions de droit à une audition équitable, de droit d'entendre la preuve contre soi et de répondre sont inexistantes. Les directives du commissaire, instructions divisionnaires et ordres permanents élaborent quelques garanties procédurales mais elles se comparent en rien à celles reconnues à un accusé lors d'un procès criminel. La Directive $n^{\circ} 213$, intitulée «Discipline des détenus ${ }^{47}$ », prévoit entre autres que le détenu a le droit de recevoir à l'avance un avis de l'infraction qui lui est reprochée, un sommaire de la preuve contre lui, le droit de comparaître en personne et de faire entendre des témoins à la discrétion du directeur.

Mais comme les directives n'ont pas force de loi, elles ne constituent qu'un guide de conduite à l'intention des officiers et des décideurs et ne peuvent conférer aux détenus des droits au sens strict du terme. En d'autres

46. Michael JACKSON, «Justice Behind the Walls - A Study of the Disciplinary Process in Canadian Penitentiary", loc. cit., note 9, p. 7.

47. D.C. $n^{\circ} 213,17$ mai 1979. 
mots, les détenus ne peuvent pas faire sanctionner le non-respect des garanties procédurales prévues dans ces directives ou exiger que les autorités s'y conforment.

Le droit dans l'institution carcérale continue, pendant cette période, à n'être qu'un instrument aux mains du pouvoir plutôt qu'un instrument de protection contre le pouvoir. La notion de droits des personnes incarcérées n'existe toujours pas et, bien que le langage employé diffère légèrement, il n'est reconnu aux prisonniers aucun droit susceptible d'être mis en œuvre par le pouvoir judiciaire (sinon celui d'être relaxé au moment prescrit par la loi) ni aucun moyen de s'opposer aux empiétements des autorités administratives. L'imprécision des termes employés dans les textes législatifs et réglementaires est volontaire : les prérogatives du pouvoir ne sont pas entamées et la discrétion peut toujours s'exercer de façon aussi incontrôlée.

\section{L'INTÉGRATION PROGRESSIVE DU DISCOURS DES DROITS ET DES EXIGENCES JURISPRUDENTIELLES AU COURS DES ANNÉES 1979 À 1992}

Au cours de ces années, les détenus canadiens ont commencé à $s$ 'adresser aux tribunaux pour faire sanctionner leurs droits fondamentaux et, plus spécifiquement, pour faire reconnaître leur droit à un traitement équitable de la part des autorités carcérales. Contraintes par les nombreuses ordonnances judiciaires rendues pendant les années 1980, les diverses autorités législatives et administratives habilitées à élaborer des normes ont commencé timidement et de façon progressive à intégrer et codifier de nouveaux standards de justice et d'équité. Le règlement sur les pénitenciers et les directives du commissaire ont été amendés à plusieurs reprises pour intégrer diverses facettes du discours des droits fondamentaux et de l'obligation d'agir équitablement, tels le droit d'être entendu (c'est-à-dire le droit de connaître la preuve contre soi et de répondre, l'accès au dossier, le droit d'être présent aux audiences et le droit à la représentation par avocat devant certaines instances décisionnelles).

Jusqu'en 1992, le législateur n'a pas été l'initiateur de l'émergence des nouvelles normes de justice carcérale. Ce sont les tribunaux qui ont joué ce rôle en exigeant la transposition dans le monde carcéral des valeurs, standards et principes du code juridique en vigueur dans la société globale.

\section{LA CONSÉCRATION LÉGISLATIVE DES DROITS DES PERSONNES INCARCÉRÉES (1992-)}

Le législateur canadien a, en 1992, procédé à une réforme majeure de la loi sur l'incarcération et la libération conditionnelle et a ainsi repris en mains son pouvoir normatif. La nouvelle loi est redevenue très détaillée, 
comme elle l'était au tout début de l'histoire des pénitenciers au Canada. Cependant, les principales normes juridiques en vigueur dans le monde pénitentiaire sont aujourd'hui d'ordre législatif plutôt qu'administratif et se retrouvent dans la loi plutôt que dans des directives administratives non contraignantes.

Dans la première partie de la Loi sur le système correctionnel et la mise en liberté sous condition, le législateur énonce tout d'abord les objectifs et les principes devant gouverner le service correctionnel dans l'exécution de ses fonctions. L'article 3 de la loi proclame que l'objet du service correctionnel est le «maintien d'une société juste, vivant en paix et en sécurité". Les moyens pour atteindre cet objectif sont l'emploi de mesures de surveillance sécuritaires et humaines de même que la mise en cuvre de programmes visant la réadaptation des délinquants et leur réinsertion sociale à titre de citoyens respectueux des lois.

L'article 4 énumère quelque dix principes devant guider le service correctionnel dans l'exécution de son mandat. On spécifie tout d'abord que la protection de la société est le critère prépondérant dans l'application du processus correctionnel (par. a), mais on ajoute plus loin que les mesures nécessaires à la protection du public doivent être les moins restrictives possibles (par. d). Plusieurs des principes énoncés visent l'accroissement de la transparence du système en favorisant l'échange d'informations avec les détenus, les victimes et les autres acteurs du système judiciaire (par. $b$, $c$, et f).

Le cinquième principe est la codification tronquée de la norme établie par la Cour suprême dans l'affaire Solosky. Dans ce jugement, la Cour avait affirmé qu'une "personne emprisonnée conserve tous ses droits civils autres que ceux dont elle a été expressément ou implicitement privée par la $\operatorname{loi}^{48}$ ». Le législateur, au paragraphe e) de l'article 4 , s'exprime de la façon suivante: "le détenu continue à jouir des droits et privilèges reconnus à tout citoyen, sauf ceux dont la suppression ou restriction est une conséquence nécessaire de la peine infligée ». À première vue, cette formulation semble plus étroite, car elle ne fait pas référence au fait que les restrictions aux droits des détenus doivent être prévues par la loi mais s'appuie sur un critère beaucoup plus vague de «conséquence nécessaire de la peine ».

Le sixième principe voulant que les décisions du service doivent être claires et équitables est également une codification de principes jurisprudentiels bien établis (par. g). Deux autres principes ne sont pas à proprement parler le résultat de l'intervention juđiciaire, mais plutôt du discours des droits et de tendances généralement admises dans la société actuelle. On prévoit ainsi que les directives d'intérêt général, les programmes et les 
méthodes doivent respecter les différences ethniques, culturelles et linguistiques ainsi qu'entre les sexes et tenir compte des besoins propres aux femmes, aux autochtones et autres groupes particuliers (par. h). Dans un autre ordre d'idées, la loi spécifie que le service doit veiller au bon recrutement du personnel et à la bonne formation de ses agents (par. j).

D'autres déclarations de principe se retrouvent ailleurs dans la loi. L'article 28, par exemple, affirme que le détenu a le droit d'être incarcéré dans le milieu le moins restrictif possible compte tenu du degré de surveillance jugé nécessaire, de la facilité d'accès à sa collectivité, à sa famille, à son milieu culturel et linguistique ainsi que de la présence de programmes lui convenant.

Le langage employé laisse souvent une large marge de manœuvre aux autorités. Ainsi, cet article 28 spécifie que le service «doit s'assurer, dans la mesure du possible, que le pénitencier dans lequel est incarcéré le détenu constitue le milieu le moins restrictif possible». Quel argument, susceptible de convaincre un tribunal ou autre, est-il possible de tirer d'une disposition qui emploie à deux reprises dans la même phrase des termes comme «dans la mesure du possible " et «le moins possible»? Une telle formulation a aussi pour effet d'individualiser les situations et d'empêcher les revendications de type collectif.

Le règlement énonce lui aussi quelques principes dont le devoir d'assurer la sécurité des détenus. L'article 83 prévoit que, dans le but d'assurer un milieu pénitentiaire sain et sécuritaire, le service doit veiller à ce que chaque établissement soit conforme aux exigences des lois fédérales en matière de santé, de sécurité, d'hygiène et de prévention des incendies et qu'il soit inspecté régulièrement par les responsables de l'application de ces lois.

De telles déclarations de principe sont les bienvenues, mais la multiplication des principes est susceptible de donner lieu a des problèmes de hiérarchisation. Le législateur a été prudent en spécifiant que la protection de la société était le critère prépondérant dans tous les cas. Les droits des personnes incarcérées viennent bien après.

En plus de ces énoncés de principe, de nombreuses dispositions consacrent les droits fondamentaux des personnes incarcérées. La plupart des articles concernant les mesures disciplinaires, les transfèrements, l'isolement préventif ou punitif, les conditions d'incarcération, les fouilles, etc., sont des intégrations par le législateur des règles jurisprudentielles concernant l'application des principes de justice fondamentale en milieu carcéral élaborées par les tribunaux au cours des quinze dernières années. 


\section{CONCLUSION}

L'historique de l'évolution des normes régissant le traitement des personnes incarcérées dans les pénitenciers canadiens démontre qu'il y a eu au cours des dernières décennies une véritable révolution et un changement de perspective radical. Pour employer la terminologie de Dworkin, nous sommes passés d'une perspective utilitariste axée sur les besoins de l'institution (goal-based theory) à une perspective de justice fondée sur les droits individuels (right-based theor $y^{49}$ ). Ce changement de perspective s'explique par plusieurs phénomènes d'ordre sociologique et juridique qui ont marqué la société nord-américaine au cours de ces années tels l'extension des libertés civiles aux groupes de moindre pouvoir et l'abandon par les tribunaux de leur attitude de non-intervention et de laissez-faire (handsoff doctrine $)^{50}$.

En énumérant les droits des détenus et en établissant plusieurs garanties procédurales, la nouvelle loi intègre le discours des droits fondamentaux et du respect de la règle de droit, de l'équité procédurale et des principes de justice fondamentale. Ces mesures législatives devraient contribuer à l'émergence d'un modèle légal plus formel et moins arbitraire, à la transparence du système et à l'encadrement du pouvoir discrétionnaire. Cependant, en choisissant d' encoder $^{51}$ » les droits des personnes incarcérées de façon assez précise, le pouvoir exécutif sera plus à même, éventuellement, de contrôler leur expansion et d'orienter le décodage des normes par les tribunaux. Ces derniers se sentiront davantage liés par cette législation détaillée et ne pourront plus tirer parti d'un silence qui n'existe plus.

49. Ronald DWORKIN, Taking Rights Seriously, Cambridge, Harvard University Press, 1977, p. 177.

50. J'ai étudié ailleurs les facteurs ayant contribué à l'émergence des normes dans le monde carcéral. Voir Lucie LEMONDE, «L'évolution des normes dans l'institution carcérale ", à paraître dans Revue canadienne Droit et SociétélCanadian Journal of Law and Society, 1995.

51. Selon l'expression de Gérard TIMSIT, Les Noms de la loi, Paris, PUF, Les voies du droit, 1991, $199 \mathrm{p}$. 